\title{
Photon and electron identification with the ATLAS detector
}

\section{Cerda Alberich* $*$}

Instituto de Fisica Corpuscular (University of Valencia - CSIC), Spain

E-mail: leonor.cerda.alberichecern.ch

\begin{abstract}
The identification of prompt photons and the rejection of background, originating mostly from photons from hadron decays, relies on the high granularity of the ATLAS calorimeter. The electron identification is based on a likelihood discriminant to separate isolated electron from background electron originating from photon conversions, hadron misidentification and heavy flavour decays. Additionally, isolation variables provide further handles to separate signal and background. The measurements of the efficiencies of the electron and photon identification and isolation selections are performed with data. Tag and probe techniques are used with $\mathrm{Z} \rightarrow \mathrm{ee}, \mathrm{J} / \psi$ $\rightarrow$ ee and $\mathrm{Z} \rightarrow 11 \gamma$ decays. Inclusive photon samples are also used to measure photon identification efficiency.
\end{abstract}

The results of these measurement with the pp collisions data recorded in 2015 at $\sqrt{s}=13 \mathrm{TeV}$, corresponding to an integrated luminosity of $3.2 \mathrm{fb}^{-1}$ are presented, as well as a first look at 2016 data.

38th International Conference on High Energy Physics

3-10 August 2016

Chicago, USA

\footnotetext{
*Speaker.

$\dagger$ On behalf of the ATLAS Collaboration.

$\ddagger$ This work is partially supported by the Spanish Ministry of Economy, Industry and Competitiveness and the European Regional Development Fund (ERDF), contract number FPA2015-65652-C4-2-R (MINECO/FEDER).
} 


\section{The ATLAS detector}

The ATLAS detector [1] is a multi-purpose apparatus with a forward-backward symmetric cylindrical geometry and nearly $4 \pi$ solid angle coverage. It consists of the following detectors: the Inner Detector (Pixel, Silicon Microstrip (SCT) and Transition Radiation Tracker (TRT)), the Lead-liquid argon electromagnetic (EM) calorimeter, the Hadronic and Forward calorimeters and the Muon system.

\section{Electron and Photon Identification}

Signal electrons are identified by different sets of likelihood-based identification criteria which are chosen to be $95 \%, 90 \%$ and $80 \%$ efficient for electrons with $E_{\mathrm{T}} \approx 40 \mathrm{GeV}$, and referred to as loose, medium and tight operating points respectively. For Run-II, several changes to the input variables used for electron identification (ID) have been introduced [2]. Figure 1 shows a schematic view of the electron reconstruction and ID:

- Taking advantage of the new innermost pixel layer, the insertable B-Layer (IBL), the number of hits in this layer is used for discriminating between electrons and converted photons.

- The change in the TRT gas led to modifications in the detector response and prompted the introduction of a new discriminating variable in the electron ID algorithms.

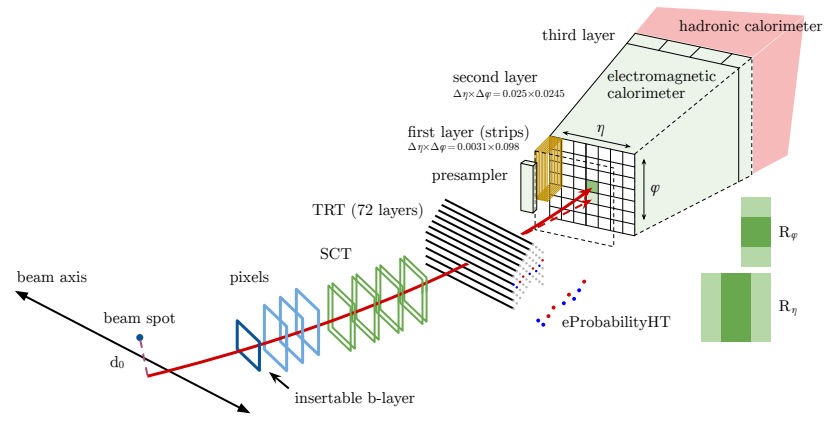

Figure 1: Schematic view of the electron reconstruction and identification [2].

The identification (ID) of prompt photons and the rejection of background coming mostly from photons from hadron decays relies on the high granularity of the ATLAS calorimeter [3]. There are two levels of ID:

- The loose ID exploits the discriminating variables (DV), such as the ratio of $E_{\mathrm{T}}$ in the hadronic calorimeter to $E_{\mathrm{T}}$ of the EM cluster or the number of hits in the Inner Detector, only in the hadronic and in the EM calorimeter second sampling layer, providing a highly efficient selection with fair background rejection, typically used for the trigger and background studies.

- The tight ID level exploits the full granularity of the EM calorimeter and applies tighter requirements also on the DVs used by the loose ID. 


\section{Electron ID efficiency measurements}

The measurement of the efficiencies of the electron identification and isolation cuts are performed with the data using tag and probe techniques with large statistics sample of $\mathrm{Z} \rightarrow$ ee and $\mathrm{J} / \psi$ $\rightarrow$ ee decays.

- This method consists on passing a strict selection on one of the electron candidates ('tag') together with the requirements of the di-electron invariant mass, which allows for a loose pre-identification of the other electron candidate ('probe').

- The low $E_{\mathrm{T}}$ range (from 7 to $20 \mathrm{GeV}$ ) is covered by $\mathrm{J} / \psi \rightarrow$ ee and suffers from a significant background contamination, while $\mathrm{Z} \rightarrow$ ee events are used for measurements above $15 \mathrm{GeV}$, as shown in Figure 2.
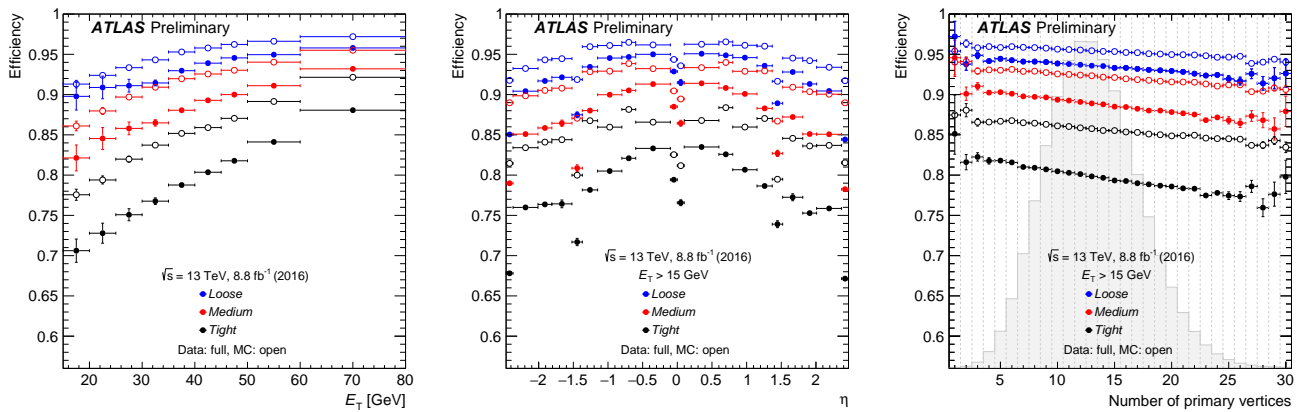

Figure 2: Electron ID efficiencies in $\mathrm{Z} \rightarrow$ ee events as a function of transverse energy $E_{\mathrm{T}}$ (left), pseudorapidity $\eta$ (middle) and the number of reconstructed primary vertices (right) [4].

\section{Photon ID efficiency measurements}

Figure 3 shows several methods that are used to measure with data the efficiency of the photon identification requirements, to cover a broad energy spectrum:

- At low energy, radiative photons from $Z \rightarrow 1 l \gamma$ decays are selected by placing kinematics requirements on the dilepton pair, on the invariant mass of the three particles in the final state and on the quality of the two leptons.

- In the medium energy range, similarities between electrons and photon showers are exploited using $\mathrm{Z} \rightarrow$ ee decays and photon ID efficiencies are obtained using a 'tag-and-probe' method.

- At high energy, inclusive photon samples are used by applying the matrix method, which classifies the photons between prompt and background photon candidates, passing or not tight ID criteria.

Figure 4 shows the difference between the simulation and the data-driven measurements, which is taken into account by computing data-to-MC efficiency ratios, also referred to as scale factors (SF). Most SF values are close to unity. This confirms that the simulation, with the applied corrections, provides a good description of the photon shower shapes in the collision data. 

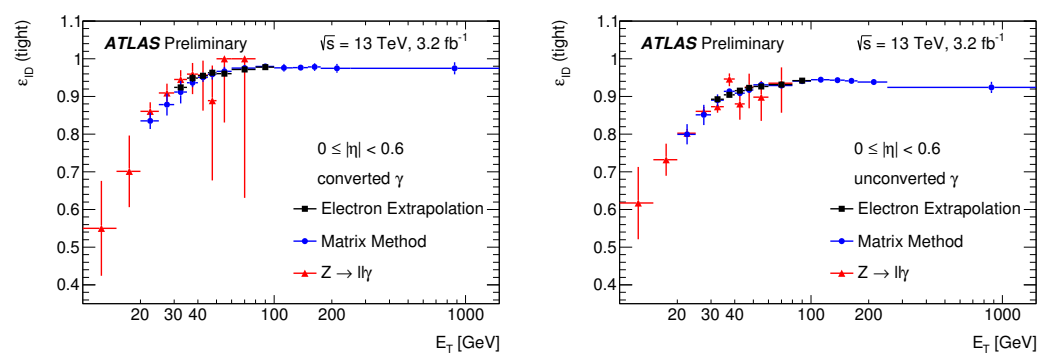

Figure 3: Comparison of the data-driven measurements of the identification efficiency for converted photons (left) and unconverted photons (right) [3].
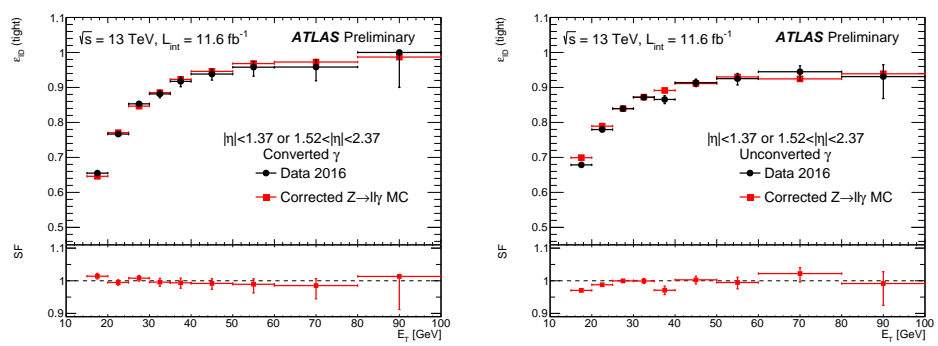

Figure 4: Comparison of the radiative $\mathrm{Z}$ boson data-driven efficiency measurements of converted (left) and unconverted (right) photons to the $\mathrm{Z} \rightarrow 1 \mathrm{l} \gamma$ simulation as a function of the $E_{\mathrm{T}}$, in the region $15 \geq E_{\mathrm{T}} \geq 100$ $\mathrm{GeV}$. The lower panel shows the ratio between the data and the simulation [5].

\section{Conclusions}

Independent analyses have been pursued to measure the photon and electron ID efficiencies.

The SF obtained for photons are closer to unity than the ones obtained for electrons because of the correction of the shower shape variables in simulation applied on photons to account for the average data-MC difference on these variables.

The electron ID efficiencies are found to be robust with respect to the number of primary vertices, in the range probed by the available data. The ID algorithms based on a multivariate likelihood discriminator have been optimised such that only a small dependence on the pileup condition remains and will be investigated in the future.

\section{References}

[1] ATLAS Collaboration, The ATLAS Experiment at the CERN Large Hadron Collider, JINST 3 (2008) S08003.

[2] ATLAS Collaboration, Electron efficiency measurements with the ATLAS detector using the 2015 LHC proton-proton collision data, ATLAS-CONF-2016-024, 2016.

[3] ATLAS Collaboration, Photon identification in 2015 ATLAS data, ATL-PHYS-PUB-2016-014, 2016.

[4] ATLAS Collaboration, Electron identification efficiency measured with $Z \rightarrow$ ee events using 2016 data, EGAM-2016-002, 2016.

[5] ATLAS Collaboration, Photon Identification Efficiencies using 2016 Data with radiative Z boson decays, EGAM-2016-003, 2016. 